\title{
Blastic Plasmacytoid Dendritic Cell Neoplasm in a Young Female: Rare and Challenging
}

\begin{abstract}
Blastic plasmacytoid dendritic cell neoplasm (BPDCN) is a rare hematopoietic malignancy with an aggressive behavior, seen commonly in the elderly age group and usually involves the skin. Evaluation, diagnosis, and treatment pose unique challenges with poor outcomes. We report a case of an 18-year-old female who presented with lymphadenopathy and bicytopenia. The diagnosis of BPDCN was an initial challenge. She thereafter received intensive chemotherapy followed by an allogenic partially mismatched sibling donor hematopoietic cell transplantation, only to relapse in a few months. Beginning from the diagnosis, the management of this rare disorder and its relapse until her last followup provided several learning opportunities.
\end{abstract}

Keywords: Blastic plasmacytoid dendritic cell neoplasm, CD123, hematopoietic cell transplant

\section{Introduction}

Blastic plasmacytoid dendritic cell neoplasm(BPDCN), formerly known as primary cutaneous natural killer/T-cell lymphoma, has now been classified as a separate entity in the 2016 World Health Organization (WHO) myeloid neoplasms classification. $^{[1,2]} \quad$ Plasmacytoid dendritic cell has been described as the cell of origin. ${ }^{[1]}$ BPDCN can be observed in all age groups, but it is more common in elders. ${ }^{[1,3,4]}$ The male-to-female ratio is $2.2: 1 .^{[4]}$ After the first case reported in 1994, around 200 cases have been described in the literature. ${ }^{[4]}$ It constitutes $0.44 \%$ of all hematologic malignancies in a year. ${ }^{[4]}$ Majority cases present with cutaneous manifestations such as plaque or nodules, red to purple in color ${ }^{[5]}$ Its presentation is often confused with other dermatologic disease manifestations. The disease also involves the bone marrow, spleen, and lymph nodes. ${ }^{[5]}$ BPDCN may coexist with myelodysplastic syndrome, and $10 \%-20 \%$ cases may progress to acute myeloid leukemia. ${ }^{[5,6]}$

\section{Case Report}

An 18-year-old adolescent female presented with bilateral glandular neck swellings of 15 days. No systemic symptoms were observed. On examination, the cervical

This is an open access journal, and articles are distributed under the terms of the Creative Commons Attribution-NonCommercial-ShareAlike 4.0 License, which allows others to remix, tweak, and build upon the work non-commercially, as long as appropriate credit is given and the new creations are licensed under the identical terms.

For reprints contact: WKHLRPMedknow_reprints@wolterskluwer.com lymph nodes were firm to hard and nontender. There was a single cutaneous maculopapular lesion, $2.5 \mathrm{~cm} \times 2 \mathrm{~cm}$ in size over left anterior aspect of the thigh, reddish-purple in color which resolved spontaneously. The liver and spleen were not palpable. Investigations showed a hemoglobin level of $7.8 \mathrm{~g} / \mathrm{dl}$, white blood cell count of $29,000 / \mathrm{cu} \mathrm{mm}$, and platelet count of $59,000 / \mathrm{cu} \mathrm{mm}$, with $68 \%$ atypical cells on peripheral blood smear [Figure 1]. Organ functions were unremarkable. Imaging identified retroperitoneal lymphadenopathy. Bone marrow aspirate and biopsy showed a hypercellular marrow with $87 \%$ blasts suggestive of acute leukemia [Figure 2]. Flow cytometric analysis revealed tumor cells to be positive for CD4, CD56, CD123, CD304 (BDCA-4), CD117, CD7, CD45, and human leukocyte antigen (HLA)-DR. The cells were negative for CD13, CD33, CD10, CD19, CD20, cMPO, TdT, CD41, and CD61 [Figure 3]. These findings were suggestive of BPDCN, although CD303 (BDCA-2) was not done due to unavailability. She received four cycles of hyper-CVAD chemotherapy regimen, and bone marrow was in morphological remission after cycle IB. She underwent allogenic hematopoietic cell transplantation (HCT) with an 8/10 HLA antigen partially mismatched (high-resolution typing) sibling

\footnotetext{
How to cite this article: Modak K, Garg JK, Mishra DK, Radhakrishnan VS. Blastic plasmacytoid dendritic cell neoplasm in a young female: Rare and challenging. Indian $\mathrm{J}$ Med Paediatr Oncol 2020;41:761-3.
}

\section{Ketan Modak ${ }^{1}$, Jeevan Kumar Garg ${ }^{1}$, Deepak Kumar Mishra², Vivek S Radhakrishnan $^{1}$}

${ }^{1}$ Department of Clinical Haematology and Bone Marrow Transplantation, Tata Medical Center, Kolkata, West Bengal, India, ${ }^{2}$ Department of Laboratory Haematology, Tata Medical Center, Kolkata, West Bengal, India

Submitted: 17-Jun-2019 Revised: 17-Sep-2019 Accepted: 14-Oct-2019 Published: 29-Oct-2020

Address for correspondence: Dr. Vivek S Radhakrishnan, Department of Clinical Haematology and Bone Marrow Transplantation, Tata Medical Center, 14, MAR (EW),

New Town, Rajarhat, Kolkata, West Bengal, India.

E-mail: drvivekradhakrishnan@ yahoo.com

Access this article online Website: www.ijmpo.org DOI: 10.4103/ijmpo.ijmpo_143_19 Quick Response Code:

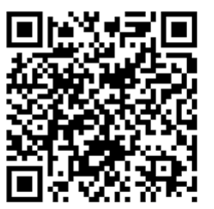




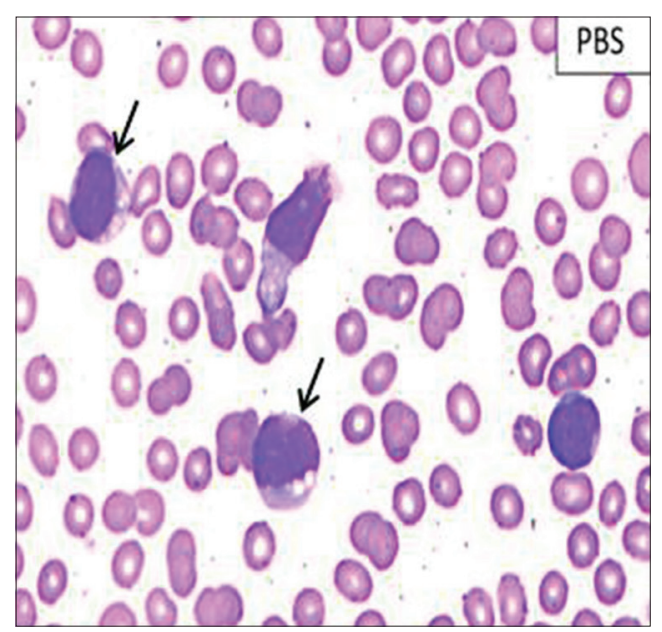

Figure 1: Peripheral blood smear showing tumor cells (marked by arrow)

sister. Standard myeloablative conditioning with total body irradiation (TBI) and injectable busulfan was administered, and CD34 cell dose of $6.0 \times 10^{6}$ cells $/ \mathrm{kg}$ (with a CD3 cell dose of $0.54 \times 10^{8}$ cells $/ \mathrm{kg}$ ) was infused. She received graft-versus-host disease (GVHD) prophylaxis with posttransplant cyclophosphamide on day +3 and day +4 along with tacrolimus and mycophenolate mofetil from day +5 onward. Neutrophil and platelet engraftment occurred on day +15 and day +16 , respectively. During her peritransplant period, she developed acute gut GVHD on day +15 which responded to standard methylprednisolone therapy. While on methylprednisolone, she had a reactivation of cytomegalovirus for which she received ganciclovir for 2 weeks followed by valganciclovir. She was on regular follow-up without significant symptoms or morbidity but developed asymptomatic medullary relapse 19 months posttransplant. Intensive treatment and alternative "palliative" options were discussed. The patient and family chose to pursue decitabine monotherapy with intent for disease control only due to financial constraints. The patient developed febrile neutropenia after two cycles of decitabine and eventually died due to intracranial hemorrhage thereafter, 24.5 months from the initial diagnosis.

\section{Discussion}

Management of this young patient provided three learning opportunities for the treating team: arriving at a diagnosis in an unusual presentation of a very rare disorder, clinical management of aggressive disease in the background of scanty literature evidence, and treatment of relapse in such a disease setting. Our patient had peripheral blood and bone marrow involvement with cervical and retroperitoneal lymphadenopathy at presentation. There was a single cutaneous lesion which resolved spontaneously before treatment. With respect to diagnosis, the 2008 WHO Classification identifies BPDCN as a neoplasm which typically expresses CD4 and CD56 in addition to at least 1 of the pDC-associated antigens - CD123, TCL-1, CD304,

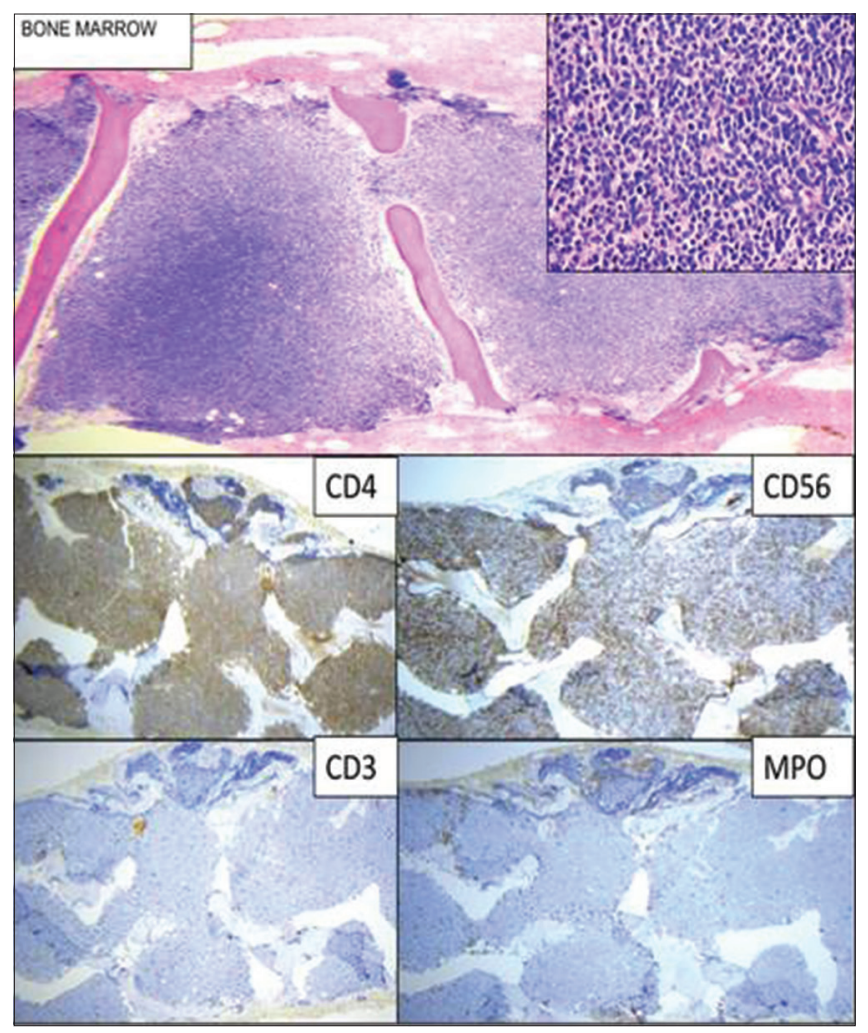

Figure 2: Bone marrow biopsy with magnified view and immunohistochemistry markers (CD3, CD4, CD56, and MPO)

or CD303/BDCA2 - in the absence of lineage-specific markers. ${ }^{[6]}$ A diagnostic criteria with 5 point scoring system based on flow cytometry has also been mentioned wherein a score of more than 2 favors the diagnosis of BPDCN. ${ }^{[6]}$

To date, there has been no consensus regarding the standard treatment of this disease. Although the disease usually shows early clinical response, relapse is very common. Various chemotherapy combinations such as acute myeloid leukemia (AML)-based induction, cyclophosphamide, doxorubicin, vincristine and prednisolone (CHOP), hyper-CVAD, and steroids have all been used. ${ }^{[5,7-9]}$ In a retrospective analysis of BPDCN patients who were treated with hyper-CVAD, CR rate was found to be $90 \%$ and OS ranged from 23 to 29 months, which was superior to a CHOP-like regimen. ${ }^{[9]}$ A recent review recommends AML like induction therapy followed by consolidation with allogenic HCT, owing to its myeloid lineage. ${ }^{[6,10]}$ HCT in first complete remission (CR1) has been suggested for a durable outcome. ${ }^{[6]}$ As per the European Society for Blood and Marrow Transplantation, there was an increase in overall survival and no relapse within 27 months in patients who received allogenic HCT in CR1. ${ }^{[10]}$ In a case series of HCT in ten patients (6 allogenic HCT and 4 autologous HCT) with a median age of 28.5 years and conditioning regimen of cyclophosphamide with TBI, overall survival was improved with the use of myeloablative conditioning followed by HCT (31.5 months) as compared to chemotherapy alone (13 months). ${ }^{[3]}$ 


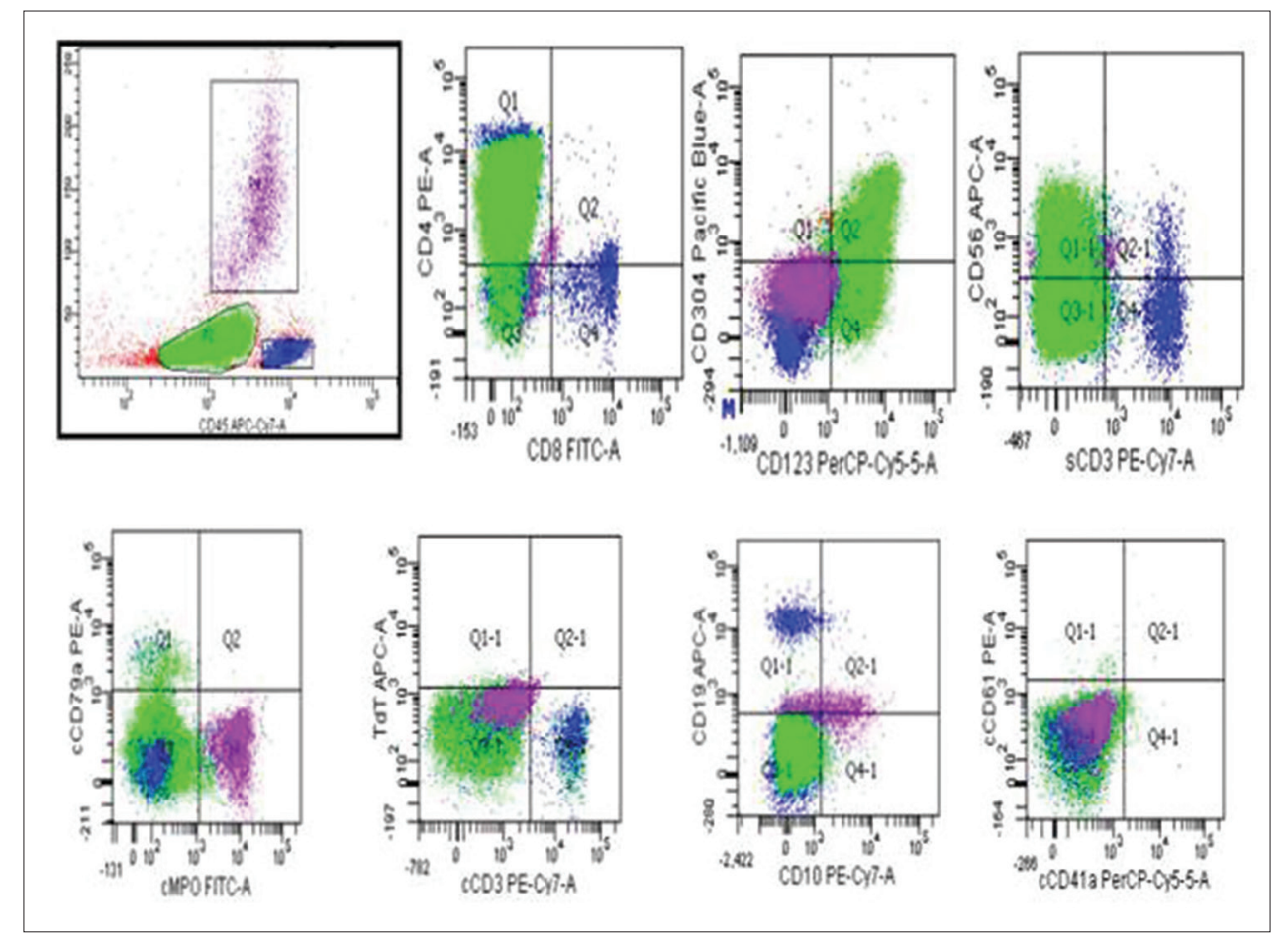

Figure 3: Flow cytometry analysis on bone marrow aspirate sample

Management of relapsed disease is also fraught with worse outcomes using conventional chemotherapy as well as newer reported therapeutic options such as hypomethylating agents, bendamustine, pralatrexate, and venetoclax ${ }^{[1]}$ It is worth noting that tegraxofusp-ezrs (ELZONRIS, a Monoclonal Ab, SL-401, directed against interleukin-3 receptor alpha chain [CD123] along with a truncated diphtheria toxin fusion protein), an immunotoxin genre of targeted immunotherapy has been recently approved by the USFDA (December 2018) with a reported CR rate of around $55 \%$ with a duration of response of around 5 months. ${ }^{[1]}$ Ongoing clinical trials evaluating novel therapeutic modalities strategies such as CAR-T cells (e.g., NCT03203369 and NCT03485547) will shed more light on the management of this aggressive malignancy ${ }^{[1]}$

BPDCN carries a poor prognosis with a median survival of around 12-16 months, and overall survival depends on the age and clinical characteristics of the patient. ${ }^{[3,4,8]}$ This case report exemplifies a rare disorder presenting at an unusual age with challenges of the diagnosis and management that are unique. It is important to initiate the treatment early and consolidate it with HCT for longer remission.

\section{Financial support and sponsorship}

Nil.

\section{Conflicts of interest}

There are no conflicts of interest.

\section{References}

1. Kerr D $2^{\text {nd }}$, Zhang L, Sokol L. Blastic plasmacytoid dendritic cell neoplasm. Curr Treat Options Oncol 2019;20:9.
2. Arber DA, Orazi A, Hasserjian R, Thiele J, Borowitz MJ, Le Beau MM, et al. The 2016 revision to the world health organization classification of myeloid neoplasms and acute leukemia. Blood 2016;127:2391-405.

3. Reimer P, Rüdiger T, Kraemer D, Kunzmann V, Weissinger F, Zettl A, et al. What is CD4+CD56+malignancy and how should it be treated? Bone Marrow Transplant 2003;32:637-46.

4. Żychowska M, Batycka-Baran A, Woźniak Z, Maj J. Blastic plasmacytoid dendritic cell neoplasm: A rare lymphoma of extremely aggressive course. Postepy Dermatol Alergol 2017;34:504-6.

5. Martín-Martín L, López A, Vidriales B, Caballero MD, Rodrigues AS, Ferreira SI, et al. Classification and clinical behavior of blastic plasmacytoid dendritic cell neoplasms according to their maturation-associated immunophenotypic profile. Oncotarget 2015;6:19204-16.

6. Sullivan JM, Rizzieri DA. Treatment of blastic plasmacytoid dendritic cell neoplasm. Hematology Am Soc Hematol Educ Program 2016;2016:16-23.

7. Feuillard J, Jacob MC, Valensi F, Maynadié M, Gressin R, Chaperot L, et al. Clinical and biologic features of CD4(+) CD56(+) malignancies. Blood 2002;99:1556-63.

8. Pagano L, Valentini CG, Pulsoni A, Fisogni S, Carluccio P, Mannelli F, et al. Blastic plasmacytoid dendritic cell neoplasm with leukemic presentation: An Italian multicenter study. Haematologica 2013;98:239-46.

9. Pemmaraju N, Thomas DA, Kantarjian H, Duvic M, Khoury JD, Patel K, et al. Blastic plasmacytoid dendritic cell neoplasm (BPDCN): A large single-center experience: Analysis of clinical and molecular characteristics and patient outcomes. Blood 2015;126:3746.

10. Male HJ, Davis MB, McGuirk JP, Abhyankar S, Aljitawi OS, Zhang $\mathrm{D}$, et al. Blastic plasmacytoid dendritic cell neoplasm should be treated with acute leukemia type induction chemotherapy and allogeneic stem cell transplantation in first remission. Int J Hematol 2010;92:398-400. 